\title{
Visual Grouping in Menu Interfaces
}

\author{
Duncan P. Brumby and Susan Zhuang \\ UCL Interaction Centre \\ University College London \\ London WC1E 6BT UK \\ Brumby@cs.ucl.ac.uk
}

\begin{abstract}
Menu interfaces often arrange options into semantic groups. This semantic structure is then usually conveyed to the user by supplementary visual grouping cues. We investigate whether these visual grouping cues actually help users locate items in menus faster, and whether there is potential for these powerful grouping cues to impede search when used inappropriately. Thirty-six participants performed known-item searches of word menus. These menus differed along three dimensions: (1) whether visual grouping cues were used, (2) whether items were semantically organized, and (3) the number of items belonging to each semantic group. Results show that the usefulness of visual grouping entirely depends on the underlying semantic structure of the menu. When menus were semantically organized, having visual grouping cues delineate the boundaries between large semantic groups resulted in the fastest search times. But when semantically unrelated items were visually grouped together, participants took far longer to locate targets. Menu designers should therefore take great care to avoid visually grouping semantically unrelated items as this has the potential to hinder menu interactions.
\end{abstract}

\section{Author Keywords}

Visual search; menus; eye-tracking; interaction science.

\section{ACM Classification Keywords}

H.5.2 User Interfaces: evaluation/methodology, screen design, theory and methods.

\section{INTRODUCTION}

Menu interfaces appeared in the earliest personal computers and are still in widespread use today. They are used in all desktop applications, on most websites, on smartphones and tablets, and even on home heating control panels and medical devices. Regardless of the application domain, menu interfaces should allow the user to locate and select the desired item as quickly and easily as possible.

\footnotetext{
Permission to make digital or hard copies of all or part of this work for personal or classroom use is granted without fee provided that copies are not made or distributed for profit or commercial advantage and that copies bear this notice and the full citation on the first page. Copyrights for components of this work owned by others than ACM must be honored. Abstracting with credit is permitted. To copy otherwise, or republish, to post on servers or to redistribute to lists, requires prior specific permission and/or a fee. Request permissions from Permissions@acm.org. CHI 2015, April 18 - 23, 2015, Seoul, Republic of Korea Copyright 2015 ACM 978-1-4503-3145-6/15/04...\$15.00 http://dx.doi.org/10.1145/2702123.2702177
}
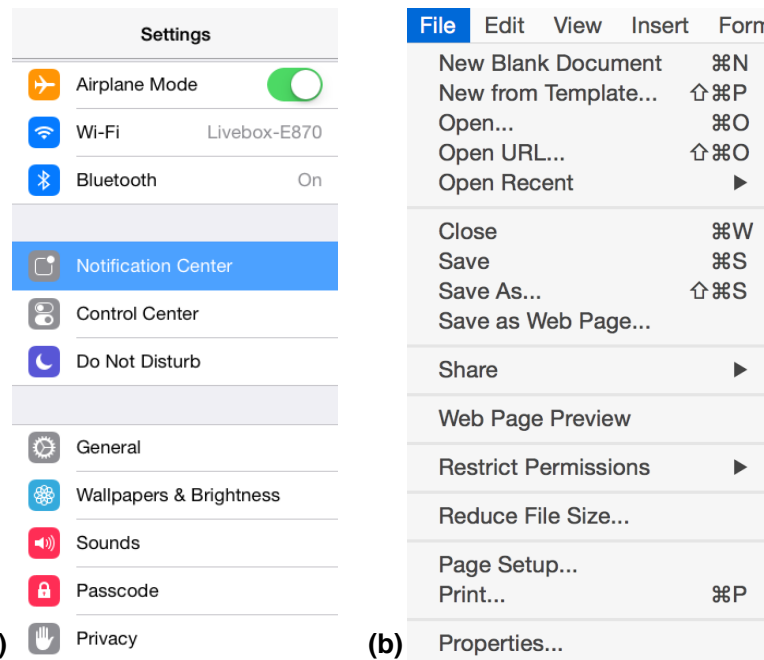

(a)

Figure 1.(a) Settings menu from Apple iPad iOS7 and (b) File menu from Microsoft Word Mac 2011. Both menus group semantically related content together and use visual grouping cues (either spatial proximity or common region) to communicate the intended structure.

An extensive literature has sought to understand the factors that influence search time during menu interactions $[2,3,4,6,7]$. One important finding is that organizing the content of menus into semantically related groups helps users locate items faster [4]. This semantic grouping effect has been replicated numerous times [2,6], and examples of semantically arranged menus abound. For instance, Figure 1 shows two common menu interfaces (the Settings menu from Apple iPad and the File menu from Microsoft Word). Both group functionally related items together (e.g., Airplane Mode, WiFi, and Bluetooth are all concerned with managing radios of the iPad, whereas the next group of items is concerned with managing notifications).

The menus shown in Figure 1 also use visual grouping cues to make the intended semantic organization of the menu pop out. The Settings menu of the iPad uses spatial proximity (i.e., spacing) to group items, while the desktop application menu bar of Microsoft Word uses common region (i.e., dividing lines). Despite the apparent widespread use of visual grouping cues in menu interfaces, we wonder whether these cues actually help users locate items faster. An early study by Parkinson, Sisson, and Snowberry [7] found that visual grouping can facilitate menu search performance. Participants searched menus that organized content into semantically related groups. Visual 
grouping cues were then used to reinforce this coherent structure, helping participants locate items faster.

In practice though it is often quite challenging for designers to get the semantic structure of a menu interface right [5]. Examples of menus that group semantically unrelated items together are surprisingly common. For instance, the final group of items in the Apple iPad Settings menu (i.e., General, Wallpaper, Sounds, Passcode, Privacy) might best be described as the 'everything else' group. Previous research has used unordered (randomized) menu layouts to capture the situation in which menu items have weak semantic congruence $[2,4,6]$, finding that users are slower at locating targets in unordered menus. Is there a danger that inappropriate visual grouping cues might compound the problems of poor semantic grouping and slow search time yet further?

A menu search experiment was designed to unpick the influence of visual grouping effects from the influence of semantic ordering effects. Participants performed knownitem searches in menus that differed in terms of: (1) whether visual grouping cues were present, (2) whether items were semantically organized, and (3) the number of items belonging to each semantic group. This last factor was included to reflect the variability that is usually seen in how items are grouped in menus (e.g., Fig. 1 shows group sizes of between one and five items).

Following Parkinson et al. [7], we would expect visual grouping cues to help participants locate targets faster when menu items are arranged into cohesive semantic groups. But when semantically unrelated items are visually grouped together we might expect search times to increase because these cues will give the impression that there is a meaningful structure to the menu (even though there is none).

In designing the experiment we sought to replicate and extend Halverson and Hornof [6]. But unlike in this previous study, we choose to use line boxes to group items (as shown in Fig. 2). Using boxes is this way is known to be a particularly effective visual grouping cue for communicating informational structure [1]. A further benefit of using boxes is that the presence/absence of visual grouping can be varied without impacting other aspects of the display. In contrast, using spatial proximity to group items would increase the physical distance between groups of items, and this is known to affect search behavior [3].

\section{METHOD}

\section{Participants}

Thirty-six participants (nine male) aged between 19 and 23 years were recruited through the UCL Psychology Subject Pool. All were native English speakers, with normal or corrected-to-normal vision, and experienced computer users.

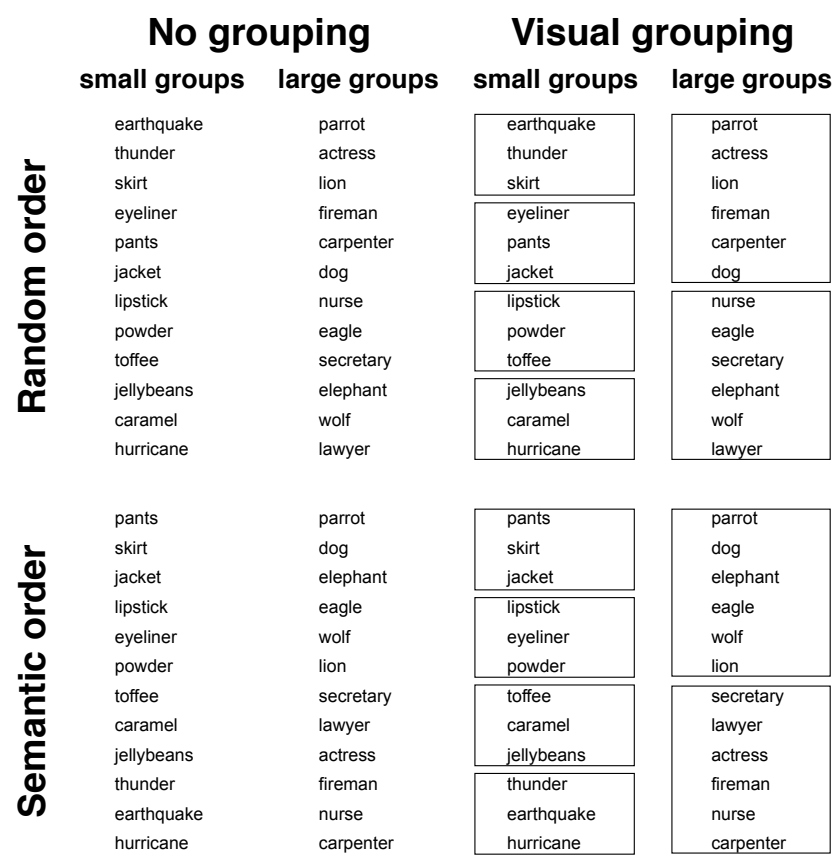

Figure 2. Illustration of each experimental condition (semantic order $\times$ semantic group size $\times$ visual grouping). Actual menus contained 3 columns of 12 menu items each, all conforming to one of the conditions depicted above.

\section{Materials}

All menus contained 36 single-word items. We generated these menus from the same database of words as used in previous studies $[2,3,6]$. This database is useful because it contains 560 unique words (with character length $M=6.2$, $S D=2.0$, range: $2-12$ ), organized into natural categories. There are 14 top-level categories (e.g., animal, building, entertainment), with each of these separated into four midlevel categories (e.g., bird, farm animal, tropical fish, and wild animal are all members of the animal category). Finally, for each mid-level category there are 10 instances (e.g., bluebird, canary, starling, eagle, hawk are all members of the bird sub-category). Human ratings have previously been gathered for these materials and confirm that items from the same semantic groups are judged as being more closely related than items from a different semantic group (see [2] for details).

Each menu contained 36 items that were arranged as three columns of 12 items each. The horizontal separation between items in each column was 30 pixels $\left(0.70^{\circ}\right.$ of visual angle), with the first item being 80 pixels from the top of the screen. The vertical separation between each column was 250 pixels ( $6^{\circ}$ of visual angle). All text was presented in an Arial font, size 10. Items had to be arranged across multiple columns (giving a 2D menu); had we presented all 36 items as a single 1D list the font would have been far smaller and items much closer together.

Stimuli were presented on a 17-inch monitor (1024 x 768 resolution). Eye movements were recorded using a Tobii 1750 eye tracker. The sampling rate of the eye tracker was $50 \mathrm{~Hz}$, with gaze point accuracy of less than $0.5^{\circ}$ of visual 
angle. The experimental software ran on Microsoft Windows XP. An optical mouse was used, set at the 'medium' speed via the system control panel.

\section{Design}

A $2 \times 2 \times 2$ (semantic order $\times$ semantic group size $\times$ visual grouping) mixed design was used. Semantic order and group size were both manipulated as within-subjects factors. In the semantic order condition, items belonging to the same group were listed together, whereas in the random condition, items were jumbled and presented in no order. Menus differed in terms of the number of items that came from the same top-level category (i.e., semantic group). Menus contained either six groups of six related items (large groups), or were made up of 12 groups of three related items (small groups). Visual grouping was manipulated as a between-subjects factor. This meant that half of the participants searched menus that always had boxes grouping sets of items, while the other half of participants searched menus that did not have boxes.

\section{Procedure}

Participants were informed that they would be required to search menus to locate a given target. At the start of each trial, participants were told what word they were looking for, as well as a top-level category description of that word (e.g., "Find a type of animal, wolf"). After reading this, participants started a trial by clicking a button on the screen. This made the target description disappear, and the menu appeared beneath it. Participants were told that there was only a single target item in each menu and that they had to locate it as quickly as possible. Participants selected an item by clicking on it with the mouse. If an incorrect selection was made, participants were instructed to make another selection from the same menu after being shown the target information again. The trial ended when the participant had successfully selected the target. Before proceeding to the next trial, participants were given feedback on the time taken to locate the target. The purpose of this feedback was to reinforce the instruction to locate the target quickly.

Each participant completed 100 search trials, divided into two blocks of 50 trials each (grouped by semantic order condition). The sequence in which each semantic order condition was experienced was counter-balanced between participants: half of the participants did all of the semantic order searches before the random order searchers, and vice versa. The presentation order of different semantic group size conditions was randomized within a given semantic order condition. The eye tracker was calibrated before the start of each block to ensure accurate gaze tracking. Participants were seated approximately $60-70 \mathrm{~cm}$ from the monitor throughout the experiment, and were given the opportunity for a short break in between blocks. The entire experiment took approximately 30 minutes to complete.

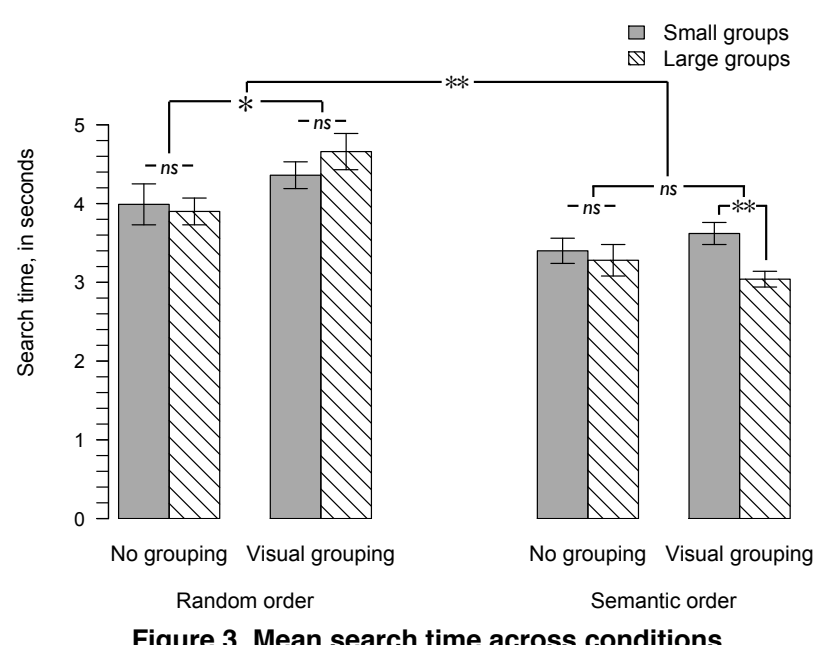

Error bars depict standard errors. $* p<.05 * * p<.001$

\section{RESULTS}

A mixed ANOVA was used, with a significance level of .05 for judging the significance of effects. We excluded trials from the analysis in which the participant did not correctly select the target on the first attempt. Accuracy was generally very high: mean accuracy for each condition was between $98-100 \%$, and data from only 44 trials were excluded (from a total of 3,600).

The primary measure of interest was search time (i.e., how long it took participants to correctly locate and select the target item). Figure 3 shows mean search time data for each condition. It can be seen in the figure that participants were significantly faster at locating targets in menus that were semantically organized $(M=3.34, S D=0.56)$ than menus that were randomly organized $(M=4.23, S D=0.80)$, $F(1,34)=79.52, p<.001, \eta_{\mathrm{p}}{ }^{2}=.70$. While there was not a significant main effect of either semantic group size or of visual grouping, $F$ 's $<2$, there was a significant three-way interaction, $F(1,34)=3.94, p=.05, \eta_{\mathrm{p}}{ }^{2}=.10$.

The significant three-way interaction suggests that the effects of semantic group size and visual grouping are dependent on whether or not the menu is semantically organized. We therefore separate the analysis of semantically organized menus from the randomly organized ones, and for each, consider the effect of semantic group size and visual grouping. For semantically organized menus, there was a significant two-way interaction between group size and visual grouping, $F(1,34)=4.23, p=.05$, $\eta_{\mathrm{p}}{ }^{2}=.11$. Follow-up tests of this interaction show that when there was visual grouping in the menu, participants were significantly faster at searching menus with larger semantic groups $(M=3.04, S D=0.41)$ than menus with smaller semantic groups $(M=3.62, S D=0.60), F(1,34)=13.78, p$ $<.001, \eta_{\mathrm{p}}{ }^{2}=.28$. However, there was no effect of group size when there was no visual grouping, $F<1$. For randomly organized menus, there was a different pattern of effects. Participants were now significantly slower at searching menus that had visual grouping cues $(M=4.51$, $S D=0.73)$ than those that did not show grouping cues $(M=3.95, S D=0.78), F(1,34)=5.00, p=.03, \eta_{\mathrm{p}}{ }^{2}=.13$. 
Finally, eye movement data are considered to understand the effects of semantic and visual grouping. In general, fixation count data revealed the same pattern of statistical effects as the search time data. This is because there is a simple linear relationship between fixation counts and search time, $R^{2}=.45, t(34)=5.41, p<.001$. A more interesting finding from the eye movement data is that the mean distance (in pixels) between contiguous fixations was greater when participants searched semantically organized menus $(M=6.66, S D=1.58)$ than when they searched randomly organized menus $(M=5.94, S D=1.41), F(1,34)$ $=18.83, p<.001, \eta_{\mathrm{p}}{ }^{2}=.36$. This suggests that participants were adapting their search strategy to the semantic structure of the menu. This makes sense: encountering a semantically unrelated item during search informs the searcher that the target is unlikely to be in that part of the menu, and that attention should be directed elsewhere, allowing the target to be located faster. There was however no effect of either grouping or semantic group size on mean distance between fixations, $F$ 's $<1$.

\section{DISCUSSION}

Consistent with previous research $[2,4,6]$, we found that organizing the content of menus into semantically related groups helped participants locate items faster. Consistent with [7], adding visual grouping cues to semantically organized menus helped further speedup search times, but only when menus contained fewer, larger groups. Being able to easily see the boundaries between semantically distinct groups presumably helped participants to systematically assess each group in turn. This meant that groups containing an irrelevant item could be dismissed, so helping participants quickly hone in on the target group.

The results of this study also highlight the potential for visual grouping cues to hinder menu interactions when used inappropriately. In particular, we found that when semantically unrelated items were grouped together, participants took far longer to locate targets. This is an important finding because in practice it is often difficult to get the semantic structure of a menu interface right [5]. Adding visual grouping cues to an already poorly organized menu can make matters worse and should be avoided.

\section{Limitations}

Menus are used in a variety of settings, by a variety of users, to achieve a variety of goals. We end by considering how some of the decisions made in designing this experiment might warrant further investigation.

First, the study reported here used line-drawn boxes to group items. There are of course other visual cues that can be used. In Figure 1a, for instance, spatial proximity is used. Color can also be used (as in [6]). It is presently not clear how the choice of visual grouping cue might influence the pattern of results reported here, although we suspect it will make little difference.
Second, participants were given a precise description of the item that had to be located in the menu (known-item search). But often the user only has a vague idea of how the target will be specified (semantic search). This distinction is interesting because users adopt different eye gaze strategies depending on the kind of activity they are engaged in [3]. We might expect visual grouping cues, which highlight the fine-grained semantic structure of the menu, to offer even greater benefit to users engaged in a semantic search (because they cannot adopt simple letter-matching strategies that would be otherwise insensitive to visual grouping effects).

Finally, we had participants interact with each menu only once. While this is representative of many infrequent or first time menu interactions, there are many occasions in which users frequently interact with the same menu. Previous research has shown that some menu layout effects are diminished when users repeatedly interact with the same menu [2]. Usually this is explained by assuming that users learn over time the spatial location of targets in the menu [2]. Would we find a similar reduction in effect size here? All of these avenues require further empirical work.

\section{ACKNOWLEDGEMENTS}

This work was supported by the UK Engineering and Physical Sciences Research Council grant EP/G059063. We thank Gilles Bailly, Antti Oulasvirta, and Andrew Howes for encouraging us to publish this work, as well as our reviewers for their constructive feedback.

\section{REFERENCES}

1. Bae, J. \& Watson, B. Reinforcing visual grouping cues to communicate complex informational structure. IEEE Transactions on Visualization and Computer Graphics, 20, (2014), 1973-1982.

2. Bailly, G., Oulasvirta, A., Brumby, D.P., \& Howes, A. Model of visual search and selection time in linear menus. Proc. CHI '14, ACM (2014), 3865-3874.

3. Brumby, D.P., Cox, A.L., Chung, J., \& Fernandes, B. How does knowing what you are looking for change visual search behavior? Proc. CHI '14, ACM (2014), 3895-3898.

4. Card, S.K. User perceptual mechanisms in the search of computer command menus. Proc CHI '82, ACM (1982), 190-196.

5. Chi, E.H., Rosien, A., Supattanasiri, G., et al. The bloodhound project: Automating discovery of web usability issues using the infoscent simulator. Proc $\mathrm{CHI}$ '03, ACM (2003), 505-512.

6. Halverson, T. \& Hornof, A.J. The effects of semantic grouping on visual search. Proc CHI EA '08, ACM (2008), 3471-3476.

7. Parkinson, S.R., Sisson, N., \& Snowberry, K. Organization of broad computer menu displays. International Journal of Man-Machine Studies, 23, (1985), 689-697. 